\title{
Review
}

\section{Genome graphs and the evolution of genome inference}

\author{
Benedict Paten, ${ }^{1}$ Adam M. Novak, ${ }^{1}$ Jordan M. Eizenga, ${ }^{1}$ and Erik Garrison ${ }^{2}$ \\ ${ }^{1}$ Genomics Institute, CBSE, 501C Engineering 2, University of California Santa Cruz, Santa Cruz, California 95064, USA; ${ }^{2}$ Wellcome \\ Trust Sanger Institute, Cambridge CB10 1SA, United Kingdom
}

\begin{abstract}
The human reference genome is part of the foundation of modern human biology and a monumental scientific achievement. However, because it excludes a great deal of common human variation, it introduces a pervasive reference bias into the field of human genomics. To reduce this bias, it makes sense to draw on representative collections of human genomes, brought together into reference cohorts. There are a number of techniques to represent and organize data gleaned from these cohorts, many using ideas implicitly or explicitly borrowed from graph-based models. Here, we survey various projects underway to build and apply these graph-based structures-which we collectively refer to as genome graphs-and discuss the improvements in read mapping, variant calling, and haplotype determination that genome graphs are expected to produce.
\end{abstract}

\section{The triumph of the human reference genome}

The sequencing of a human genome was truly a landmark achievement (International Human Genome Sequencing Consortium 2001). Over a number of years, the genome assembly has steadily improved (International Human Genome Sequencing Consortium 2004; Church et al. 2011) to the point that the current Genome Reference Consortium (GRC) human genome assembly, GRCh38 (Schneider et al. 2017), is arguably the best assembled mammalian genome in existence, with just 875 remaining assembly gaps and fewer than 160 million unspecified " $\mathrm{N}$ " nucleotides (as of GRCh38.p8).

Perhaps one reason the reference genome has been so effective as an organizing system is that the average human is remarkably similar to it. From short read-based assays, it is estimated that the average diploid human has between 4.1 and 5 million point mutations, either single nucleotide variants (SNVs), multinucleotide variants (MNVs), or short indels, which is only around 1 point variant every $1200-1450$ bases of haploid sequence (The 1000 Genomes Project Consortium 2015). Such an average human would also have about 20 million bases ( $\sim 0.3 \%$ of the genome) affected by around 2100-2500 larger structural variants (The 1000 Genomes Project Consortium 2015). Both these estimates are likely somewhat conservative as some regions of the genome are not accurately surveyed by the short-read technology used. Indeed, long-read sequencing demonstrates an excess of structural variation not found by earlier short-read technology (Chaisson et al. 2015; Seo et al. 2016).

\section{Reference allele bias}

Despite the relative effectiveness of the reference as a coordinate system for the majority of the genome, there is increasing concern that using the human reference as a lens to study all other human genomes introduces a pervasive reference allele bias. Reference allele bias is the tendency to underreport data whose underlying
DNA does not match a reference allele (Degner et al. 2009; Brandt et al. 2015). This bias arises chiefly during the read mapping step in resequencing experiments. In order to map correctly, reads must derive from genomic sequence that is both represented in the reference and similar enough to the reference sequence to be identified as the same genomic element. When these conditions are not met, mapping errors introduce a systematic blindness to the true sequence.

In the context of genetic variant detection, this problem is most acute for structural variation. Entirely different classes of algorithm are required to discover larger structural variation simply because these alleles are not part of the reference (Sudmant et al. 2015). Furthermore, the numerous large subsequences entirely missing from the reference in turn surely contain population variation (Sudmant et al. 2015). Describing these variants and their relationships is simply not possible with the current reference model. Reference allele bias also has the potential to affect some genetic subpopulations and some regions of the genome more than others, depending on the ancestral history of the reference genome at each locus.

We believe reference allele bias is driving the field of genome inference in two directions. First, with improvements in sequencing technology, the field is beginning to use unbiased de novo assembly to make inferences on individual samples. (For a glossary of terms used in this review, see Table 1.) Second, the field is developing richer reference structures that more completely represent the variation present within the population. As a substrate for variant detection, these reference structures should mitigate reference bias by permitting a fuller complement of read mappings (see "Genome inference with genome graphs"). These directions are not mutually exclusive; hybrid approaches will ultimately be desirable. However, this perspective focuses on reference structures, particularly for human genomics. In particular, we show how human reference-assisted variant calling is naturally progressing toward graph-based reference structures.
Corresponding author: benedict@soe.ucsc.edu

Article and publication date are at http://www.genome.org/cgi/doi/10.1101/ gr.214155.116. Freely available online through the Genome Research Open Access option. (c) 2017 Paten et al. This article, published in Genome Research, is available under a Creative Commons License (Attribution 4.0 International), as described at http://creativecommons.org/licenses/by/4.0/. 
Table 1. A glossary of terms used in this perspective

\begin{tabular}{|c|c|}
\hline Pangenome & $\begin{array}{l}\text { A collection of genomic sequences that are analyzed together or used as a reference (Computational Pan-Genomics Consortium } \\
\text { 2016). }\end{array}$ \\
\hline Repeatome & The repetitive portion of a genome or pangenome. \\
\hline Scaffold & A sequence where some of the characters are uncertain or unknown. \\
\hline Homomorphism & An embedding of one graph into another graph. \\
\hline Monoallelic & $\begin{array}{l}\text { A conceptual model of genomic variation that highlights the presence or absence of pieces of sequence, or of connections } \\
\text { between those pieces. }\end{array}$ \\
\hline Directed graph & A graph, made up of a set of nodes and a set of edges, where the edges are ordered pairs of a from node and a to node. \\
\hline Bidirected graph & A graph, made up of a set of nodes and a set of edges, where the edges are unordered pairs of (node, orientation) tuples. \\
\hline Biedged graph & $\begin{array}{l}\text { A graph, made up of a set of nodes and a set of edges, where the edges come in two types. Black edges are directed and labeled } \\
\text { with a sequence, while gray edges are undirected. }\end{array}$ \\
\hline Genome graph & A graph used to represent a pangenome. \\
\hline Sequence graph & $\begin{array}{l}\text { A genome graph in which the nodes are labeled with nucleotide sequences. Generally bidirected, but with an equivalent biedged } \\
\text { representation. }\end{array}$ \\
\hline Collapsed graph & $\begin{array}{l}\text { A genome graph in which sequences that were represented by different nodes in a previous graph are now represented by a } \\
\text { single node. }\end{array}$ \\
\hline Tip & A side of a node in a bidirected graph that has no edges connected to it. \\
\hline Ultrabubble & $\begin{array}{l}\text { As shown in Figure 3, a minimal directed acyclic subgraph within a sequence graph that has no tips and is connected to the rest } \\
\text { of the graph through two nodes: a source on the left and a sink on the right (Paten et al. 2017). }\end{array}$ \\
\hline Walk & A sequence of oriented visits to nodes in a graph, describing a traversal that is consistent with the structure of the graph. \\
\hline Path & A walk in which no node is visited more than once; sometimes used in the literature to mean just "walk" (Garrison et al., in prep.). \\
\hline HMM & $\begin{array}{l}\text { A Hidden Markov Model, which is a directed graph where the nodes are states, which emit symbols from a distribution, and the } \\
\text { edges are transitions between states, which are chosen probabilistically. }\end{array}$ \\
\hline PRG & A Population Reference Graph, which is a type of HMM-based pangenome (Dilthey et al. 2015). \\
\hline Variant discovery & $\begin{array}{l}\text { The process of finding novel genomic variants that may be present in an individual but which are not present in a database of } \\
\text { known variation. }\end{array}$ \\
\hline Variant decision & The process of determining the genotype of an individual, drawing from a predetermined set of possible states. \\
\hline $\begin{array}{l}\text { Genome } \\
\text { inference }\end{array}$ & $\begin{array}{l}\text { The process of estimating the genome of an individual or set of individuals from a combination of observed sequencing data for } \\
\text { the individual(s) in question and prior information about the population(s) that produced the individual(s). }\end{array}$ \\
\hline $\begin{array}{l}\text { De novo } \\
\text { assembly }\end{array}$ & $\begin{array}{l}\text { Creating a set of scaffolds describing a genome from a set of input reads or other information, by combining observed sequences } \\
\text { together, without using a previous set of scaffolds or genomic reference. Often uses graph-based intermediates, such as de } \\
\text { Bruijn graphs. }\end{array}$ \\
\hline
\end{tabular}

\section{Richer reference structures}

The original reference human genome assembly was essentially a monoploid representation (International Human Genome Sequencing Consortium 2001). The primary goal was to produce a single representative sequence albeit with regions of uncertainty-that is, a single "scaffold" - for each physical chromosome. It also included a handful of alternate scaffolds representing allelic variation, but they had no formalized relationship to the main scaffold. Recognizing that some highly polymorphic regions of the genome were particularly poorly represented by a single reference sequence, a formal model to introduce representative alternate versions of highly variable regions was added starting with GRCh37 (Church et al. 2011). Sequences in the form of kilobase to multi-megabase "alternate locus scaffolds" were described relative to the "primary" (monoploid) assembly, anchored to locations along the primary scaffolds. In the current assembly (GRCh38.p9), these cover 178 regions and total 261 sequences.

To better represent human diversity, we might imagine creating a "reference cohort" - that is, in place of a single reference genome, a set of sequences that includes all common variation. However, representing such a cohort in the existing alternative locus scaffold system would present significant challenges. To include all alleles down to a frequency of $1 \%$ would require alternative locus scaffolds covering the entire primary reference genome, with hundreds of such sequences overlapping each genomic location.

Although such a reference cohort is already achievable and, to some extent, derivable from public data sets like the 1000 Genomes Project (The 1000 Genomes Project Consortium 2015), representing it as a collection of alternative locus scaffolds appears impractical. First, the existing primary reference genome is a poor coordinate space with which to describe the other genomes. Much large structural variation is not adequately described by the coordinates provided by the primary reference. Indeed, this is already a problem with existing alternative loci scaffolds. Second, the alternative loci model fails to capture the fine-grained homology relationships between all the sequences (Fig. 1A). For example, when mapping a new sample into a cohort, a typical sequencing read may map equally well to many equivalent subsequences in the cohort, but this ambiguity would be illusory. Rather, this multimapping is indicative of the extensive latent structure within any nontrivial reference cohort. Each subsequence actually represents the same underlying allele. We submit that, although other models can approach it to varying degrees, this latent structure is naturally represented as a mathematical graph.

\section{Genome graphs}

Graphs have a longstanding place in biological sequence analysis, in which they have often been used to compactly represent an ensemble of possible sequences. As a rule, the sequences themselves are implicitly encoded as walks in the graph. This makes graphs a natural fit for representing reference cohorts, which are by their nature ensembles of related sequences (Fig. 1B).

Perhaps the simplest common graph representation is the directed graph, in which directed walks encode a nucleotide sequence. In the context of genome assembly, de Bruijn graphs (de Bruijn 1946; Pevzner et al. 2001; Zerbino and Birney 2008) are popular directed graph representations in which each node represents a $k$-mer (a unique string of length $k$ ), and each directed edge represents an overlap of $k-1$ bases between the suffix of the "from" node and the prefix of the "to" node (Fig. 2A). De Bruijn graphs are a restricted class of vertex-labeled directed graphs, which are graphs whose nodes are labeled such that a directed walk can be

\section{Genome Research}

www.genome.org 
A

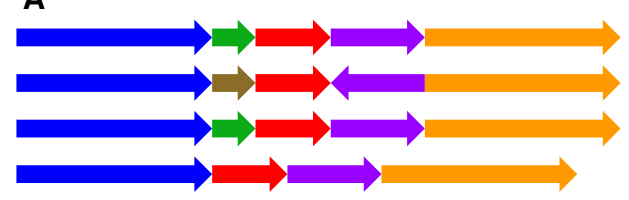

B

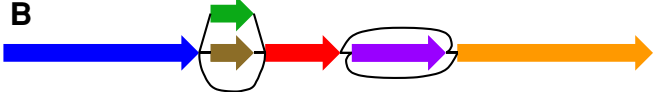

Figure 1. Schematic representation of two population-level reference structures. (A) A reference cohort, in which there is no attempt to identify homologies between the genome sequences. (B) A genome graph, in which homologies are collapsed and included as alternate paths in the graph.

interpreted as a DNA sequence, defined by the sequence of node labels along the walk (Fig. 2B). Alternatively, edge-labeled directed graphs are possible, in which case the nodes, rather than the edges, can be viewed as representing the intersection points between connected subsequences (Dilthey et al. 2015).

In either edge- or vertex-labeled representations, directed graphs do not fully express the concept of strand. That is, they do not distinguish between reading a DNA molecule in its forward and reverse complement orientations. To express strandedness, directed graphs can be generalized to bidirected graphs (Edmonds and Johnson 2003; Medvedev and Brudno 2009), in which each edge endpoint has an independent orientation, indicating whether the forward or the reverse complement strand of the attached node is to be visited when entering the node through that endpoint of the edge (Fig. 2C). Inversions, reverse tandem duplications, and arbitrarily complex rearrangements are expressible in the bidirected representation. Such complex variation cannot be expressed in the directed graph version without creating independent forward and reverse complement nodes and storing additional information to describe this complementarity. The edge-labeled version of bidirected graphs, which we call biedged graphs (Fig. 2D), give an equivalent representation.

We call a bidirected graph in which each node is labeled with a nucleotide string a "sequence graph" (Novak et al. 2017; E Garrison, J Sirén, AM Novak, G Hickey, JM Eizenga, ET Dawson, W Jones, OJ Buske, MF Lin, B Paten, et al., in prep.). In a sequence graph, a DNA sequence is read out by concatenating the node-oriented labels of a walk that always enters and exits each node through edge endpoints with opposite orientations. Labels are oriented such that entering through one endpoint orientation encodes the reverse complement of entering the node through the opposite endpoint orientation.

\section{Graphs as spatial frameworks}

Although sequence graphs are an effective way to compactly represent a cohort of genomes, they introduce some complications. One of these is that it is no longer trivial to define a locus on the reference. For a linear reference, it is sufficient to refer to a genetic element by its coordinate and extent along the reference sequence, but graphs admit multiple paths that may have complex relationships to each other (Paten et al. 2014). To overcome this issue we need to define a correspondence between structures in the graph and elements in the genome. In doing so, the genome graph becomes a spatial framework for organizing and comparing a population of genomes. Developing an effective spatial framework requires attending to several considerations regarding coordinates, alleles, ordering in graphs, and genome embedding, which we explore below.

\section{Coordinate systems}

Genome graphs need a system to refer to specific positions on the sequences they contain. Ideally, such a coordinate system should convey relevant information. The Computational Pan-Genomics Consortium (2016) has identified desirable properties of the linear reference genome model that more general spatial frameworks should attempt to preserve. These include the notions that the genome graph coordinates of successive bases within a genome should be increasing, that coordinates should be compact and human interpretable, and that bases physically close together within a genome should have similar coordinates. On top of these properties of "monotonicity," "readability," and "spatiality," Rand et al. (2016) add a further distinction between "vertical spatiality" of bases that are allelic variants of one another and "horizontal spatiality" of bases that can appear together within a single molecule. They compare two coordinate systems based on paths through a graph: one that fulfills spatiality, and another that does not fulfill spatiality but is stable under edits to the graph.

\section{Allelism in graphs}

Many genome inference tasks involve calling alleles at sites, so genome graphs must have an operational definition of a site. Some proposals seek to derive sites from a coordinate system. One option is to fall back on the linear reference's coordinate system and maintain a strict correspondence between positions in the graph and reference coordinates (Dilthey et al. 2015). However, this is somewhat restrictive, possibly nullifying some of the benefits that we are seeking from genome graphs in the first place. Another proposal uses a hierarchical, recursive approach to bolt on alternative alleles to the existing reference genome system (Rand et al. 2016), which is potentially a great improvement but still has an arbitrary dependence on the existing linear coordinates.

A more graph-centered approach is to define sites based on motifs in the genome graph. In particular, it has been proposed that sites could be described with a motif called a "superbubble" (in a directed graph) (Onodera et al. 2013) or "ultrabubble" (a

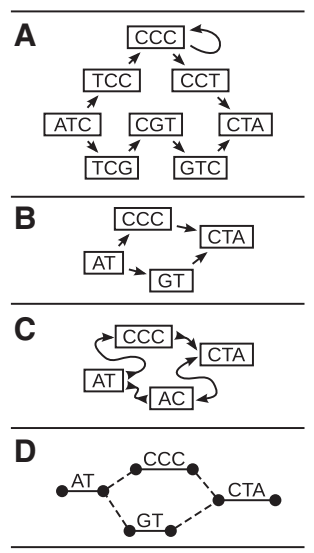

Figure 2. Four types of genome graphs, all constructed from the pair of sequences ATCCCCTA and ATGTCTA. (A) De Bruijn graph. (B) Directed acyclic graph. (C) Bidirected graph (a.k.a., sequence graph). (D) Biedged graph (a.k.a., biedged sequence graph). 
generalization to bidirected graphs) (Paten et al. 2017). In brief, ultrabubbles and superbubbles are directed acyclic subgraphs that connect to the rest of the graph through one source node and one sink node (Fig. 3). This motif tends to be created when new variants are added to a graph. Both superbubbles and ultrabubbles can also identify nesting and overlapping relationships involving structural variants, which makes them a more expressive definition of a site than the reference coordinate. Paten et al. (2017) show how this site nesting is naturally described by a cactus graph, a structure that globally organizes the sites without the need for any existing reference genome. However, this approach is incomplete-not all variation is nicely partitioned into these bubbles. Moreover, it lacks the appealing simplicity of linear reference coordinates.

\section{Pangenome ordering}

Although genome graphs do not in general provide a natural, simple coordinate system, it is possible to impose a linear coordinate system by constructing a comprehensive linear ordering of the nodes (Fig. 4; Nguyen et al. 2015). Such a structure has been described as a pangenome (Herbig et al. 2012; Nguyen et al. 2015). For any chromosome, such a complete ordering is potentially far more inclusive than any individual extant haplotype, in that it can include all the elements present in the population. As an actual nucleotide sequence (typically after collapsing substitutions into their major allele) this sort of linearized sequence can also potentially be closer to being a median of the genomes it represents. Nguyen et al. (2015) demonstrated useful properties of such an ordering for common bioinformatics tasks such as read mapping. They also illustrated how a linear pangenome can be used to more inclusively visualize a set of genomes. In addition, linear orderings of genome graphs potentially have utility for creating accessible storage (ensuring good data colocalization for adjacent graph elements) (Haussler et al. 2017) and for algorithms that need to efficiently address contiguous subgraphs of a larger genome graph.

\section{The repeatome}

A significant fraction of a typical human genome is composed of highly repetitive satellite arrays (Manuelidis and Wu 1978; Willard and Waye 1987). Highly repetitive sequences are particularly prevalent in biologically important centromeric regions (Levy et al. 2007; Miga 2015), and one of the most fundamental biological structures, the ribosome, is encoded in repetitive sequence (Levy et al. 2007; Miga 2015). We need effective tools to deal with these repetitive regions-collectively, the "repeatome" - if we are to gain a full understanding of human genomics (Miga 2015).

Genome graphs can potentially allow the repeatome, not currently meaningfully accessible with short-read sequencing approaches, to be analyzed in some fashion by collapsing repeats together in the graph (Paten et al. 2014). Rather than representing

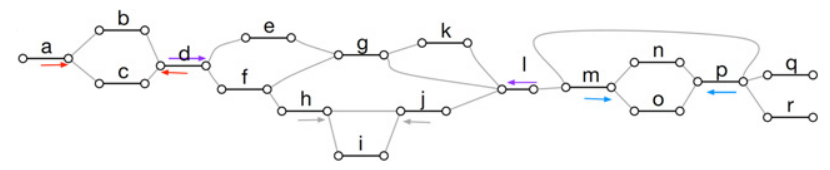

Figure 3. Ultrabubble sites in a biedged sequence graph. Each arrow shows the terminal node of a site. The color of the arrows indicates the node pairing. Note that the ultrabubble denoted by the gray pair of arrows is nested within the ultrabubble denoted by the purple arrows. (Reprinted from Paten et al. 2017, with permission from the author.)

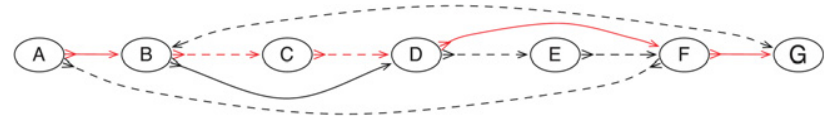

Figure 4. A pangenome ordering on a graph constructed from two genomes. The red edges indicate the path of the pangenome through the graph. The solid and dotted edges indicate the adjacencies between nodes in the two source genomes. (Adapted from Nguyen et al. 2015, with permission from the author.)

a repetitive region directly, the graph reference would represent the space of instances of a type of repeat across regions and individuals. In such a graph the presence of a specific repeat and its copy number can be identified by unique read mappings, but not necessarily the identity and locations of individual instances of the repeat-frequently a much more difficult problem.

Such a graph could be part of a larger graph reference, or it could serve as a special-purpose structure for repeat-specific studies. The technique could be applied to tandem repeat arrays as well as to more accessible isolated instances of repetitive elements. In the pioneering work of Miga et al. (2014), for example, probabilistic, condensed graphs for the $\mathrm{X}$ and $\mathrm{Y}$ centromeric repeat arrays were constructed, in which similar instances of a repeat were combined within the same graph node (Fig. 5). These graphs were then used to generate linear reference sequences for the two arrays (Miga et al. 2014), using a Markovian traversal, and have subsequently been used to define linear representations of the centromeres that are included in GRCh38 (Schneider et al. 2017).

\section{Hierarchy}

Extending the idea of collapsing repetitive sequences, the work of Paten et al. (2014) showed that it is not necessary to pick a single genome graph to act as a reference. Rather, they argued that a hierarchy of graphs related by graph homomorphisms (a projection function from the nodes of one graph to the nodes of a more collapsed representation of that graph) could be constructed in which progressively more collapsed versions of the same underlying set of genomes could be constructed and related (Fig. 6). In the most collapsed graph in the hierarchy, repetitive sequences might be fully collapsed, whereas the least collapsed graph in the hierarchy might represent the input set of haplotypes as disjoint sequences. Intermediates in this hierarchy could represent a more typical monoallelic representation of the genomes. The constructed hierarchy would have the property that mapping a subsequence to an element in a graph in the hierarchy automatically implies the mapping of the subsequence to all the more collapsed versions of that element in the more collapsed graphs in the hierarchy. In this way, mapping a sequence to a specific repeat instance would also identify the sequence as mapping to the canonical copy, classifying it as an instance of the repeat type.

\section{Haplotype embedding}

The number of paths through a genome graph increases combinatorially with the number of alternate alleles it includes. However, many of these sites are in tight linkage disequilibrium, so the number of paths that actually occur in the population increases much more slowly (Fig. 7). It can be useful for read mapping and variant calling to distinguish between paths that have actually been observed and others that are likely rare or absent in the population. One solution is to store allele frequency and linkage information in the genome graph by embedding a population cohort as a set

\section{Genome Research}

www.genome.org 
(1) HOR Array Sequence Database to Catalogue Sequence Variants

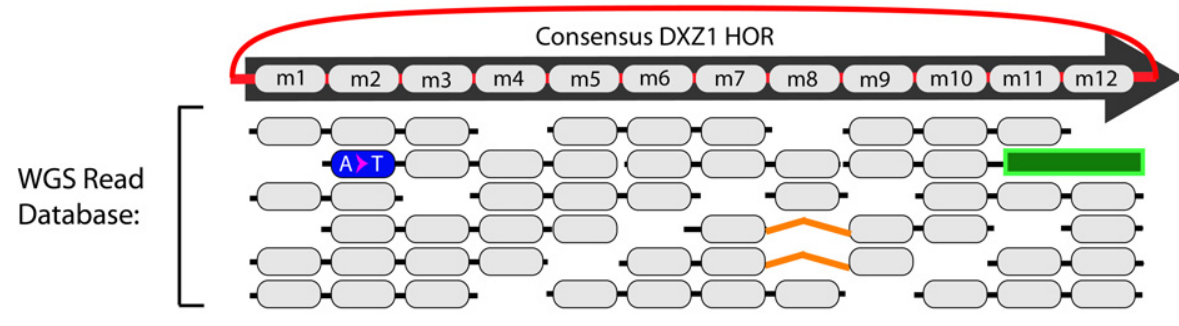

(2) Reformat into Array Sequence Graph

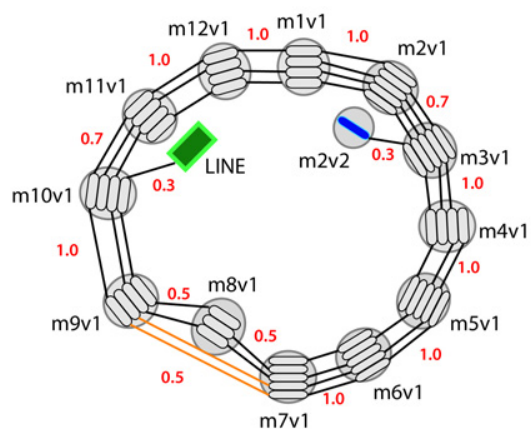

Figure 5. A schematic example of an "Array Sequence Graph" of the type used to construct a linearization of the DXZ1 repeat array in the X Chromosome centromere (Miga et al. 2014). A collection of reads (top) shown in the context of a consensus higher-order repeat are converted into a graph representation (bottom). A cycle around the graph represents a higher-order repeat, and the individual repeat units (oblongs) are represented within each node (circles). Edges between individual repeat units represent phasing information from input reads. Transitions between nodes are annotated with probabilities. (Adapted from Miga et al. 2014, with permission from the author.)

of walks through the graph, each of which represents a haplotype. Such a sequence graph with embedded walks is known as a "variation graph." This is also an instance of the graph hierarchies described above in which there is homomorphism from the population cohort onto a constructed genome graph.

A major challenge to implementing embedded haplotypes is making the data structure for storing the cohort compact enough to fit in memory while also keeping the data available for computation. In linear references, the Positional Burrows-Wheeler Transform (PBWT) was developed in response to this challenge. The PBWT is a transform of a matrix of binary haplotypes that is highly compressible and supports efficient haplotype search queries even when compressed (Durbin 2014). Building on this idea, the recently developed graph-PBWT (gPBWT) is a similar succinct data structure that supports simultaneous compression and efficient haplotype queries on haplotypes embedded as walks within a variation graph (Novak et al. 2016). It appears practical to store entire population cohorts for thousands of genomes in such an auxiliary structure, which is already implemented within the $\mathrm{xg}$ software (https://github.com/vgteam/xg).

\section{Read mapping in genome graphs}

One of the most important use cases for a genomic reference is as a target for read mapping.

Read mapping tools usually rely on one of two indexing approaches, in order to quickly find the best mapping locations for a read in a reference. Some tools, like MOSAIK, use $k$-mer-based indexing, in which short $k$-mer sequences from the reference are related to their locations using a hash table or other traditional key- value data structure (Lee et al. 2014). Other tools, such as BWA-MEM, use Burrows-Wheeler Transform-based approaches, in which the reference is stored in a succinct self-indexed data structure optimized for substring search (Li 2013). However, these approaches have traditionally been implemented against monoploid, linear reference genomes, resulting in a mapping task in which each read is placed at one or more linear coordinates. Mapping against more complex, nonlinearized pangenomes presents unique challenges.

Several notable read mapping tools (or more integrated tools that internally map or process reads) are described in Table 2. Both linear reference-based and graph-based tools are represented.

\section{Alt-aware mapping}

The current human genome assembly, GRCh38 (Schneider et al. 2017), contains alternative loci that provide different versions of sequences already represented in the primary assembly (see the section "Richer reference structures"). Mappers which can make sense of these additional representations, either as special linear sequences or as components of a graph, are marked as "alt-aware" in Table 2.

Although alt-awareness naturally falls out of many graph-based pangenome representations, it is also possible to achieve in a linear framework. BWA-MEM is one example of a linear reference-based, alt-aware mapping tool ( $\mathrm{Li}$ 2013; Church et al. 2015). Although not formally described in the literature, the alt-aware feature of BWA-MEM uses a two-step process (https://github.com/lh3/bwa/blob/1f99921b73237203e 5772bee5a8c7a254c6bcbce/README-alt.md). In the first step, reads are mapped to the primary and alt sequences, as normal, but with special rules for mapping quality calculation and primary secondary supplementary status assignment. In the second step, alignments between alt and primary sequences are used to project alignments into the primary sequence space and to update mapping qualities in light of how the primary and alt sequences fit together. This approach produces alignments to GRCh38, in the traditionally used primary sequence coordinate space, but making some use of the alt loci; there is some preliminary evidence to suggest that the resulting alignments may be better than those obtained using BWA-MEM on only the primary sequences.

If one of a person's two haplotypes in a region is much closer to an alt sequence than the primary sequence, the projection onto the primary sequence's coordinate space will at best lose information and at worst interfere with variant calling. One feature of BWA-MEM's alt-aware mode is that it also outputs alignments in the coordinate spaces of the alts, to allow variant calling in alt coordinates. However, how to use these alt-coordinate-space alignments to produce a combined set of variant calls across the linear coordinate spaces most appropriate for an individual is still an open question (see Dilthey et al. 2015). 


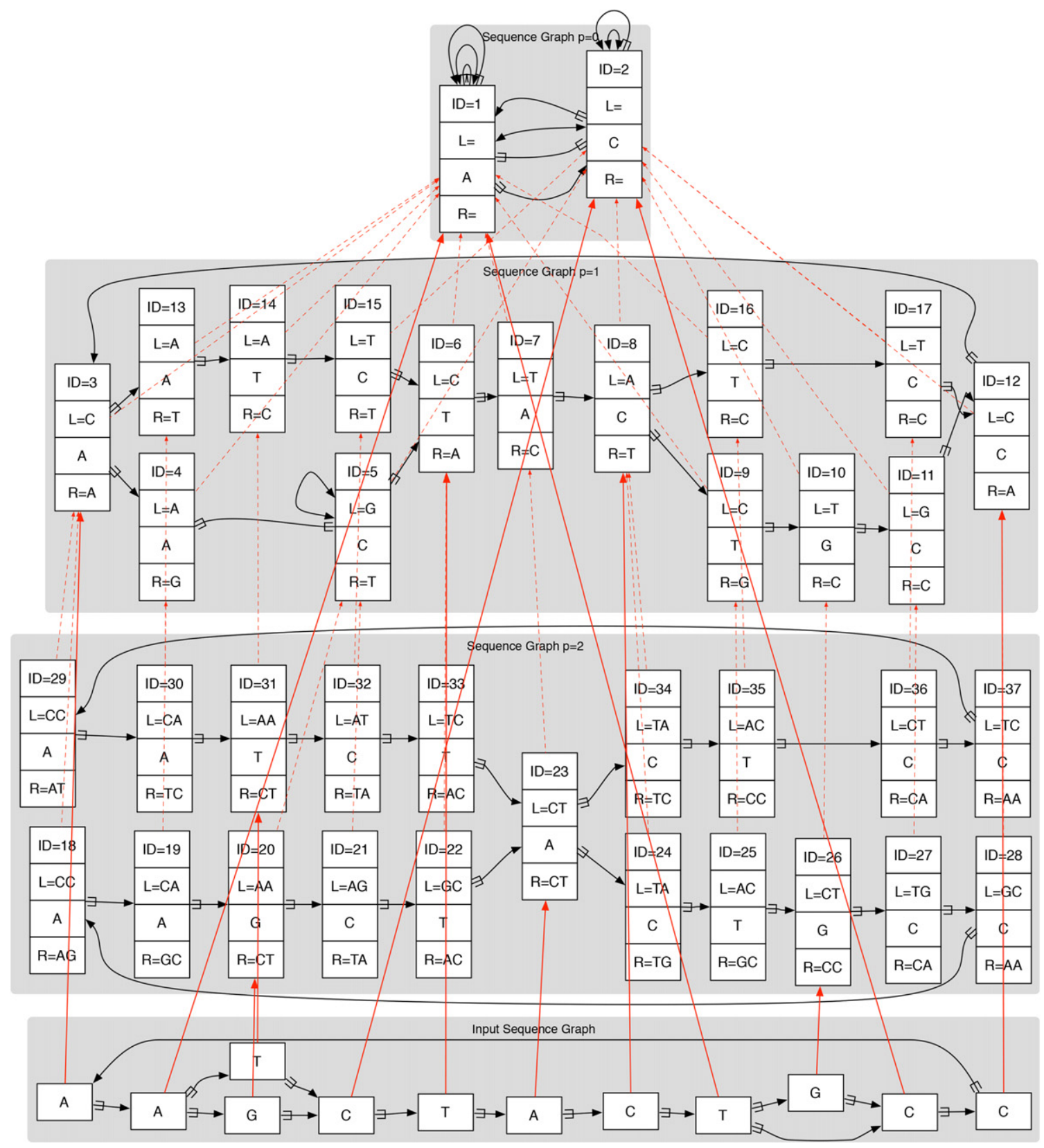

Figure 6. A reference genome graph hierarchy (most collapsed graph at the top, less collapsed lower), with an input graph (bottom) mapped to it. All the graphs in the reference hierarchy are de Bruijn graphs. Dotted red lines show projections between graphs in the hierarchy, whereas solid red lines show mapping of the input sequence graph into the hierarchy. Here, each node has a unique ID, and the $L$ and $R$ strings represent flanking contexts mapping strings required for unique identification. (Reprinted from Paten et al. 2014, with permission from the author.)

\section{Extending mapping to genome graphs}

Some graph-based approaches, like Gramtools (Maciuca et al. 2016), admit only certain highly structured graphs. This allows them to be "variant-aware" - to account for small-scale variants when computing read alignments-with a controlled amount of additional complexity over linear reference-based approaches. Other approaches can handle more general directed acyclic graphs, or even bidirected, cyclic sequence graphs. Extending local alignment, indexing, and distance measurement to graphs-and especially to complex graphs-has proven to be a challenge, and the tools in Table 2 have approached these problems in various ways.

\section{Local alignment}

Although extending fully ordered dynamic programming algorithms to partially ordered directed acyclic graphs is relatively simple (Lee et al. 2002), sequence graphs are not restricted to directed acyclic structures. Some biological structures, such as inversions, duplications, or highly variable copy number, might most naturally be represented using the bidirectedness of the sequence graph formulation, or by adding cycles to the reference. In vg, one of the tools that supports these more complex structures, cyclic bidirected graphs are "unrolled" and "unfolded" to make directed acyclic graphs that are amenable to partial-order alignment, with the 


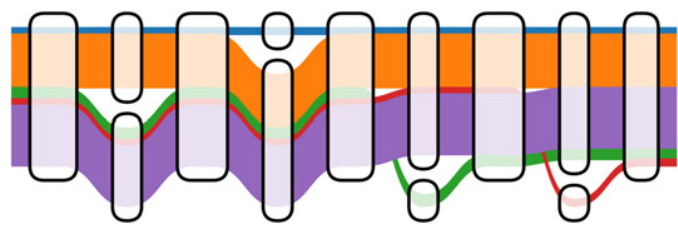

Figure 7. Distinct 1000 Genomes Project haplotypes embedded within a variation subgraph. Haplotypes are shown as colored ribbons with width proportional to the log of their frequency. The number of possible paths traversing left to right is 16 , but only five are observed in the 1000 Genomes Project because of linkage disequilibrium. (Figure based on prototype by W Beyer, pers. comm.)

results transformed back into the original graph's coordinates (Fig. 8; E Garrison, J Sirén, AM Novak, G Hickey, JM Eizenga, ET Dawson, W Jones, OJ Buske, MF Lin, B Paten, et al., in prep.). In deBGA, another such tool, complex structures in the de Bruijn graph index are flattened out when alignments are articulated against individual linear haploid assemblies (Liu et al. 2016).

\section{Indexing}

When working at the scale of whole genomes, the problem of extending indexing strategies to graphs becomes very important. Read mapping tools need to narrow down the reference genome when mapping a read, usually to one or a few regions containing candidate hits. As with tools for mapping to linear genomes, sequence graph mapping tools can be divided into those that use succinct self-index approaches and those that use $k$-mer lookup table approaches. On the succinct self-index side, one notable exam- ple is Gramtools's vBWT, in which the graph itself is represented as a modified BWT (Maciuca et al. 2016). This approach is quite elegant, but it limits the structure of the graph to one that merely represents successive sets of alternatives. On the other end of the spectrum, vg, which uses the GCSA2 graph indexing library, is able to represent arbitrary sequence graphs in its index, although index size grows combinatorially with local graph complexity (Sirén 2017; E Garrison, J Sirén, AM Novak, G Hickey, JM Eizenga, ET Dawson, W Jones, OJ Buske, MF Lin, B Paten, et al., in prep.). One interesting approach to controlling index size is illustrated in HISAT 2 (Pertea et al. 2016); although its approach is not yet described in the literature, the tool is built around a collection of small graph self-indexes, rather than a single large index.

One of the most interesting graph-based tools using a $k$-merbased index is the "population reference graph" (PRG) method of Dilthey et al. (2015). This tool uses a hidden Markov model (HMM), with emission distributions over sets of $k$-mers. The $k$ mers found in the reads are used to infer a pair of representative linear haploid genomes for a sample, denoted as paths through the HMM. These sequences are then used for realignment with the succinct self-index-based BWA backtrack, in order to produce a final set of read alignments. More broadly, de Bruijn graph-based tools, such as deBGA, are marked as using $k$-mer-based indexes, because the nodes in a de Bruijn graph are identified and looked up by $k$-mers (Liu et al. 2016).

\section{Distance measurement}

Finally, there is also the question of distance measurement in graphs, for the purposes of paired-end resolution. A serious contender in the read aligner space must deal with paired-end reads

Table 2. A comparison of various read mapping and processing tools

\begin{tabular}{|c|c|c|c|c|c|c|c|}
\hline Tool & Type & Index & $\begin{array}{l}\text { Genome- } \\
\text { scale }\end{array}$ & $\begin{array}{l}\text { Graph- } \\
\text { based }\end{array}$ & $\begin{array}{l}\text { Variant- } \\
\text { aware }\end{array}$ & $\begin{array}{c}\text { Alt- } \\
\text { aware }\end{array}$ & References \\
\hline BGREAT & Mapper & $k$-mer & $\bullet$ & $\bullet$ & $\bullet$ & $\bullet$ & Limasset et al. (2016) \\
\hline BlastGraph & Mapper & $k$-mer & & $\bullet$ & $\bullet$ & $\bullet$ & Holley and Peterlongo (2012) \\
\hline Bowtie 2 & Mapper & BWT & $\bullet$ & & & & Langmead and Salzberg (2012) \\
\hline BWA-MEM & Mapper & BWT & $\bullet$ & & & $\bullet$ & Li (2013) \\
\hline BWBBLE & Mapper & BWT & $\bullet$ & & $\bullet$ & & Huang et al. (2013) \\
\hline deBGA & Mapper & $k$-mer & $\bullet$ & $\bullet$ & $\bullet$ & $\bullet$ & Liu et al. (2016) \\
\hline GenomeMapper & Mapper & $k$-mer & $\bullet$ & - & $\bullet$ & & Schneeberger et al. (2009) \\
\hline Glia & Mapper & None & $\bullet$ & $\bullet$ & $\bullet$ & & https://github.com/ekg/glia \\
\hline Gramtools & Mapper, caller & BWT & $\bullet$ & $\bullet$ & $\bullet$ & & Maciuca et al. (2016) \\
\hline Graphite & Inference & None & $?^{\mathrm{a}}$ & $\bullet$ & $\bullet$ & & https://github.com/dillonl/graphite \\
\hline GSNAP & Mapper & $k$-mer & $\bullet$ & $\bullet b$ & $\bullet$ & & Wu and Nacu (2010) \\
\hline HISAT 2 & Mapper & BWT & $\bullet$ & & & & $\begin{array}{l}\text { Pertea et al. (2016); https://github. } \\
\text { com/infphilo/hisat2 }\end{array}$ \\
\hline iBWA & Mapper & BWT & $\bullet$ & & & $\bullet$ & $\begin{array}{l}\text { http://gmt.genome.wustl.edu/ } \\
\text { packages/ibwa/ }\end{array}$ \\
\hline MOSAIK & Mapper & $k$-mer & $\bullet$ & & & & Lee et al. (2014) \\
\hline mrsFAST-Ultra & Mapper & $k$-mer & $\bullet$ & & $\bullet$ & & Hach et al. (2014) \\
\hline PRG & Inference & $k$-mer & & - & $\bullet$ & $\bullet$ & Dilthey et al. (2015) \\
\hline SRPRISM & Mapper & Undocumented & $\bullet$ & & & $\bullet$ & $\begin{array}{l}\text { ftp://ftp.ncbi.nlm.nih.gov/pub/ } \\
\text { agarwala/srprism }\end{array}$ \\
\hline $\mathrm{vg}$ & Mapper, caller & BWT & $\bullet$ & - & $\bullet$ & $\bullet$ & Garrison et al. (in prep.) \\
\hline
\end{tabular}

Tools come in several types, noted in the "Type" column. "Mapper" tools perform read mapping; "Caller" tools also perform variant calling; "Inference" tools take in reads and perform specialized or integrated genome inference tasks. The "Index" column notes the indexing technology used by each tool. Tools are also graded on the presence or absence of several traits. "Genome-scale" tools have been demonstrated on or are marketed as suitable for the analysis of entire human genomes. "Graph-based" tools make use of an internal graph representation of variation or support adjacency information. "Variant-aware" tools are capable of accounting for point or small-scale variation in a reference. "Alt-aware" tools are capable of accounting for larger-scale replacements or for the presence of complete secondary contigs in a reference. Note that, among the tools described here, graphbased tools are always variant-aware, and only graph-based tools are both variant-aware and alt-aware.

${ }^{a}$ No concrete usage examples are provided.

${ }^{\mathrm{b}}$ Supports a splice site database. 
A

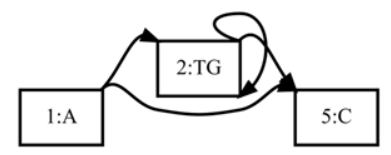

B

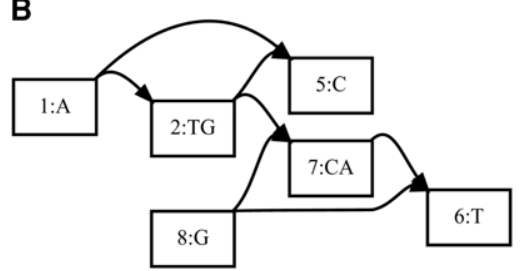

Figure 8. Bidirected sequence graph $(A)$ being unfolded into a directed acyclic graph $(B)$, in preparation for partial-order alignment. Node 6 is a reversed view of node 1 , node 7 is a reversed view of node 2 , and node 8 is a reversed view of node 5. (Reprinted from Garrison et al. [in prep], with permission from the author.)

in a reasonable way, which is challenging in a genome graph because calculating the distances between mappings, or even the relative orientations of mappings, is no longer trivial.

There are two major approaches described in the literature: doing paired-end resolution in the space of some (potentially inferred) linear sequence, and doing paired-end resolution using a graph-based distance metric. The PRG system and deBGA do paired-end resolution in the space of individual sequences: the generated pair of sequences used for BWA realignment in PRG, and the linear reference sequences embedded in the de Bruijn graph in deBGA (Dilthey et al. 2015; Liu et al. 2016). A graph distance metric is used for paired-end resolution in $\mathrm{vg}$, which can serve as an example of that approach, although the implementation does not currently consider the relative orientations of paired reads (E Garrison, J Sirén, AM Novak, G Hickey, JM Eizenga, ET Dawson, W Jones, OJ Buske, MF Lin, B Paten, et al., in prep.). Some projects, like HISAT 2, have not yet documented how their paired-end distance calculations are performed in a graph, whereas other projects, like Gramtools, do not yet implement paired-end alignment (Maciuca et al. 2016). Overall, paired-end resolution in graph reference structures is a relatively open problem.

\section{Context mapping}

In addition to graph-based mapping methods based on traditional dynamic-programming alignment, there has also been interest in alternative notions of mapping, building on the notion that a position in a sequence graph reference can have different semantics than a position in a linear reference. Precedent for this idea is found in the notion of flanking sequences for SNPs in dbSNP (Sherry et al. 2001). Originating before the first release of the human genome assembly, dbSNP was not designed around a linear reference sequence. Rather than requiring variation to be submitted by coordinate in a reference genome, variation originally instead had to be submitted along with flanking sequence, describing the context in which the variation was observed by the submitting laboratory (Sherry et al. 2001). These observed flanking sequences (perhaps obtained by Sanger sequencing), rather than a VCF position, defined the variant (Sherry et al. 2001).

In a sequence graph, the sort of offset-based coordinates that are commonly used in linear references can become unwieldy. It may be convenient to instead think of graph position as not de- fined by a coordinate but rather by a context. Instead of finding the coordinates at which a read maps and then considering a string-to-string alignment, one can assign each position in a read to a position in a sequence graph, based on correspondences between their contexts. One particular formulation of this idea, "context schemes," defines a mathematical formalism of nonredundant context assignment that ensures unambiguous mapping of individual positions and demonstrates a heuristic, potentially practicable algorithm on linear references (Novak et al. 2015). Work dealing with nontrivial graphs includes the "fuzzy context-based search" approach of Leonardsen (2016) and the small sequence graph reference structure examples of Paten et al. (2014) (for an example, see Fig. 6). It is anticipated that further research in this area could yield useful practical implementations, because the correspondence between context and position identity in a graph is quite natural. However, sensitivity challenges remain: Concepts of spatial closeness, even between adjacent positions, are difficult to translate into a context-based framework, so reads that must be placed by integrating information across the read sequence present a particular challenge.

\section{Genome inference with genome graphs}

The primary advantage of resequencing with a reference genome as opposed to de novo assembly is that it greatly simplifies the process of genome inference. While assembly needs to discover the entire genomic sequence, reference-based resequencing only needs to discover a sample's differences from the reference. Intuitively, genome graphs should provide even further advantages of the same kind. The graph contains not only the sample's approximate sequence, but also many of its specific variants. This simplifies much of the genome inference process from discovery to decision.

Despite these theoretical advantages, research on variant calling using genome graphs is still relatively nascent. In contrast, many successful methodologies have been published for calling variants using the linear reference genome (Nielsen et al. 2011). In order to be useful in practice, genome graphs must be able to translate their promised reduction in reference bias into measurable improvements in variant calling over established methodologies. Accordingly, developing variant calling algorithms for genome graphs is an important research frontier.

The leading linear reference-based variant calling tools in use today are all based on probabilistic models of sequencing data (Nielsen et al. 2011). This approach has several advantages. Modern sequencing technologies all attempt to quantify the uncertainty in their base calls. Probability models provide a natural framework to incorporate this uncertainty into genotype calls, and they allow algorithms to estimate uncertainty about genotype calls for downstream analyses.

The actual probability models used in these tools vary, but they share a common Bayesian structure. For a genotype $G$ and a set of read data $D$, the posterior likelihood of a genotype call is $P(G \mid D) \propto P(D \mid G) P(G)$. The first factor, $P(D \mid G)$, is based on a generative model of the read data. The second factor, $P(G)$, is the prior probability of a genotype, which can be flat or based on population information.

\section{Genotype likelihoods in genome graphs}

In genome graphs, genotyping consists of two distinct tasks: decision between the variants present in the graph structure a priori and discovery of novel variation. These tasks require different

\section{Genome Research}

www.genome.org 
inference processes, so it is not surprising that every technique we are aware of uses a different strategy for each.

Of these tasks, the genotype decision process is more novel to genome graphs. Recall that genome graphs are constructed so that a haplotype can be represented as a walk through the graph, modulo a few rare or private variants that are in the haplotype but not in the graph. Thus, for a diploid sample, we can essentially frame the genotype decision process as choosing two walks through the graph that contain the sample's variants, possibly phasing arbitrarily in the process (Fig. 7). This naturally leads to a path-based methodology for variant calling.

Several path-based variant calling methods have already been developed. PRG uses an HMM to compute the maximum likelihood pair of paths through its graph (Dilthey et al. 2015). BayesTyper generates local paths through nearby variants. In both cases, the genotype likelihood is based on the $k$-mer content of the reads. Both $k$-mer methods forgo read mapping, which makes them computationally efficient and avoids some conceptual ambiguities with aligning to graphs. However, $k$-mer models are also inherently susceptible to sequencing errors. The PRG group has also developed a specialized genotyping algorithm for the HLA locus that is based on read alignments, called HLA*PRG (Dilthey et al. 2016). This algorithm has high accuracy, but its computational demands are too high to scale the approach genome-wide. Finally, the vg suite includes a nascent variant calling tool that is primarily site based instead of path based. It defines sites with ultrabubbles and then defines the alleles as the set of paths through the ultrabubble. vg calls variants from read alignments with a count-based heuristic, which takes advantage of vg's flexible aligning capabilities but currently lacks a sophisticated error model.

Compared to genotype decision, the variant discovery process in graphs more closely resembles traditional genome inference. In fact, PRG and BayesTyper both use existing variant calling tools to discover variants. BayesTyper adds the discovered variants to the graph as candidates for the path-based inference, whereas PRG converts its maximum likelihood paths into candidate linear reference sequences upon which to discover novel variants; vg uses read pileups from its alignments to augment the graph so it can then use the same model for genotype discovery as decision.

In all cases, these tools sidestep some of the difficulties in generalizing the read mapping-based models that have been successful with linear reference genomes. PRG and BayesTyper both avoid mapping reads to a graph reference by using $k$-mers. HLA*PRG restricts its focus to a small section of the HLA locus. The current version of vg uses heuristics in place of a true probability model for its mapped reads. We expect that true generalizations of traditional models will be a significant advancement for the field, particularly for segregating structural variants. The fact that sequence graphs make it possible to align and model known structural variants in the same manner as other types of variation may obviate the need for separate algorithms from SNV callers (Medvedev et al. 2009).

\section{Haplotype priors on genome graphs}

As graph-based genome inference matures, it will be necessary to develop effective population priors. This need is perhaps more urgent with graphs than with linear references, because as described above (see "Haplotype embedding"), graphs can admit many paths that are not biologically meaningful. However, the novel path-based methodologies described above also represent a clear opportunity. Since the paths already combine information across sites, they provide a natural setting for incorporating linkage disequilibrium information into the prior, which is then a prior over haplotypes rather than genotypes. Doing so could mitigate the effect of the nonbiological paths and perhaps even improve the accuracy of genome inference. Basic research in this vein is already emerging. A graph generalization of the $\mathrm{Li}$ and Stevens population model using the gPBWT described above has been developed ( $\mathrm{Li}$ and Stephens 2013; Rosen et al. 2017). These or similar techniques could provide the infrastructure for efficient computation of population haplotype priors.

\section{Future opportunities and challenges}

We foresee reference cohorts replacing linear references, and that graphical models of reference cohorts will replace linear sequence assemblies as the space in which genomics is done. In the short term, some of the benefits of a more comprehensive reference cohort can be gained by resequencing against the full GRCh38 assembly, using software like BWA-MEM that properly handles alternative loci. We believe that extending existing software to fully support the alternative locus sequences of GRCh38 will lay the groundwork for full sequence graph support in the future.

Over the long term, we anticipate the development of an official reference cohort and graph-based reference that embeds this cohort. We imagine regular releases that progressively add to this structure, and that this will be overseen by an independent group, like the Genome Reference Consortium. Such an official graph could provide a truly universal coordinate system encompassing not only a single linear assembly but also the global stock of variant information from projects like the 1000 Genomes Project. Although there are benefits to having tailored approaches for specific applications, we believe that, on balance, a greater degree of standardization would be a boon in this area. Currently, too much effort is duplicated in having each group build their own partial graph-based compendia of human variation. We believe strongly in the benefits of having a concrete, comprehensive genomic data structure upon which to base our discourse. Accordingly, there is a need to generate some consensus about the best method for constructing genome graph references.

Moreover, we expect and hope for community convergence on a universally applicable graph formalism and exchange format. We anticipate that fully bidirected sequence graphs will be the ultimate winner, because of their ability to represent inversions, duplications, and translocations in a way that is natural and that accounts for gene flow in and out of such structures (Navarro et al. 1997). However, we anticipate that, in an official human sequence graph reference, these more general features would be used sparingly, for describing large-scale rearrangement events, because it is often convenient to work with graphs that are locally directed and acyclic. Moreover, a large fraction of variation is amenable to a directed acyclic representation. Standardizing on an exchange format for graphs, meanwhile, would allow hard-won insights about how to store, retrieve, and process graph data at species scale to make their way into new software from the reference interface inwards.

Perhaps the biggest challenge in migration to genome graph references, beyond the development of the necessary data science and technology, is the inertia that has developed around the 
current human reference assembly. As such a linchpin data structure, a tremendous amount of tooling and data rely upon the current reference. We advocate the development of production quality toolkits for genome graphs, that much like HTSlib and SAMtools (Li et al. 2009) for working with the current reference, make it easy to work with genome graph technology. We hope vg will become such a toolkit.

Toolkits will facilitate the transition, and coupled with a progressive, incremental approach, make adoption significantly more likely. We recognize that just as previous reference genome assemblies tend to hang around, so will the current reference human genome assembly, even in a future where a significant fraction of work has switched over to using reference genome graphs. We anticipate the need to maintain translation between linear reference assemblies and genome graphs, particularly the need for tools to lift over annotations between existing assemblies and genome graphs, just as we do today between updated assembly releases.

In the area of genome resequencing, the trend is, as always, toward longer read sizes (Jain et al. 2015), cheaper sequencing (Jain 2015), and larger read separations (Coombe et al. 2016; Putnam et al. 2016). We anticipate that these advances will allow de novo assembly to become a more practical resequencing analysis approach, and overall will increase the accessibility of largescale structural variants. Although the importance of a reference for ordering and orienting short reads may wane as long-read technology improves, the importance of a reference compendium of structural variation will increase as such variation becomes more detectable. Furthermore, the importance of a reference that provides information on known variable bases will increase as higher read-error-rate sequencing methods become more widely used.

In the area of read alignment, we recommend more research into alternative problem formulations, rather than just straightforward extensions of read mapping to graphs. Instead of finding a single best alignment of a read to a graph, for example, it would be useful to be able to obtain a collection or distribution of such alignments, which would account for different possible paths in the graph from which the read may have been generated. When there are multiple decision points in an alignment, and especially when reads are long, a single "mapping quality" value may no longer be a sufficient description of alignment confidence. Carrying more detailed uncertainty information about read mapping through to the variant calling or genome inference step of a resequencing pipeline should improve overall performance. It would also be useful to explore the idea of context-based mapping more thoroughly; we anticipate that effort spent developing more practical and efficient implementations of the basic idea would not be wasted.

Future genome inference methodologies must capitalize on these developments and translate them into improved accuracy. We have already suggested some directions for this research. Inference models that have already proven successful on linear genomes should be generalized to graphs, and future models should take advantage of new path-based variant calling methodologies to incorporate linkage disequilibrium into the population prior. Ultimately this will result in the combining of phase imputation and variant inference into one model and optimization process, allowing sampling from the posterior of genomes rather than simply genotypes. When these techniques mature, we expect that they will outperform existing genome inference tools, especially in cases involving structural variation and high polymorphism.
Moreover, the transition from discovery to decision processes will require less from the data to infer common variants. Thus, these methods will also serve the economic imperative of maintaining accuracy while reducing coverage requirements. This will in turn enable projects at larger scales. Conversely, we believe that there is more useful information to be extracted from existing sequencing samples, and that existing short-read technologies may yet be used to generate significantly more comprehensive genome inference when compared against a comprehensive genome graph. In making a cost-benefit analysis of sequencing technologies, we believe the development of reference genome graphs and large population cohorts, essentially as a better prior for inference, may significantly alter the calculation.

In this perspective, we have focused on genome graphs and their likely impact on genome inference and genetic discourse. However, it is important to recognize that genome graphs could have a similarly dramatic impact on transcriptomics and epigenetics. Although beyond the scope of this discussion, the natural extension of the reference genome to incorporate a more complete map of variation opens the possibility of directly linking transcript expression and epigenetic marks with specific alleles, thus creating an integrated structure for relating these other omics types to underlying genomic variation.

The developments described in this review indicate a rapidly growing ecosystem of tools and methods for genome inference using graph-based reference structures. As a whole, graph-based genome inference promises to mitigate the problem of pervasive reference bias, through effective incorporation of reference cohort information. This will have an impact throughout genomics, as previously intractable forms of genetic variation become assayable with the efficiency of routine resequencing experiments. For some time, researchers and ethicists have warned against the healthcare disparities that basing genomic studies on European populations could cause (Need and Goldstein 2009). Now evidence is accumulating that such disparities are already occurring in personalized medicine (Petrovski and Goldstein 2016). Reducing reference bias is an important step toward remedying this problem, and graph genomes are the most promising proposal to do so.

\section{Competing interest statement}

The authors of this manuscript include authors of the vg (E Garrison, J Sirén, AM Novak, G Hickey, JM Eizenga, ET Dawson, W Jones, OJ Buske, MF Lin, B Paten, et al., in prep.) and Glia tools described above.

\section{Acknowledgments}

We thank Karen Miga and Wolfgang Beyer for providing help and figures. We thank David Haussler, Richard Durbin, Gil McVean, and members of the reference-genomes task team of the Global Alliance for Genomics and Health for many useful discussions. We thank Daniel Zerbino and the two other anonymous reviewers for their insightful comments and feedback. We thank Glenn Hickey, Charles Markello, Yohei Rosen, Eric Dawson, Mike Lin, and all contributors to the vg software package, which is driving much of our work forward. This work was supported by the National Human Genome Research Institute of the National Institutes of Health under Award Number 5U54HG007990 and grants from the W.M. Keck foundation and the Simons Foundation. The content is solely the responsibility of the authors

\section{Genome Research}

www.genome.org 
and does not necessarily represent the official views of the National Institutes of Health.

\section{References}

The 1000 Genomes Project Consortium. 2015. A global reference for human genetic variation. Nature 526: 68-74.

Brandt DY, Aguiar VR, Bitarello BD, Nunes K, Goudet J, Meyer D. 2015. Mapping bias overestimates reference allele frequencies at the HLA genes in the 1000 Genomes Project Phase I Data. G3 (Bethesda) 5: 931-941.

Chaisson MJ, Huddleston J, Dennis MY, Sudmant PH, Malig M, Hormozdiari F, Antonacci F, Surti U, Sandstrom R, Boitano M, et al. 2015. Resolving the complexity of the human genome using singlemolecule sequencing. Nature 517: 608-611.

Church DM, Schneider VA, Graves T, Auger K, Cunningham F, Bouk N, Chen HC, Agarwala R, McLaren WM, Ritchie GRS, et al. 2011. Modernizing reference genome assemblies. PLoS Biol 9: e1001091.

Church DM, Schneider VA, Steinberg KM, Schatz MC, Quinlan AR, Chin CS, Kitts PA, Aken B, Marth GT, Hoffman MM, et al. 2015. Extending reference assembly models. Genome Biol 16: 13.

Computational Pan-Genomics Consortium 2016. Computational pan-genomics: status, promises and challenges. Brief Bioinform bbw089.

Coombe L, Warren RL, Jackman SD, Yang C, Vandervalk BP, Moore RA, Pleasance S, Coope RJ, Bohlmann J, Holt RA, et al. 2016. Assembly of the complete Sitka spruce chloroplast genome using 10X Genomics' GemCode sequencing data. PLOS ONE 11: e0163059.

de Bruijn NG. 1946. A combinatorial problem. Koninklijke Nederlandse Akademie v. Wetenschappen 1: 758-764.

Degner JF, Marioni JC, Pai AA, Pickrell JK, Nkadori E, Gilad Y, Pritchard JK. 2009. Effect of read-mapping biases on detecting allele-specific expression from RNA-sequencing data. Bioinformatics 25: 3207-3212.

Dilthey A, Cox C, Iqbal Z, Nelson MR, McVean G. 2015. Improved genome inference in the MHC using a population reference graph. Nat Genet 47: 682-688.

Dilthey AT, Gourraud PA, Mentzer AJ, Cereb N, Iqbal Z, McVean G. 2016. High-accuracy HLA type inference from whole-genome sequencing data using population reference graphs. PLoS Comput Biol 12: e1005151.

Durbin R. 2014. Efficient haplotype matching and storage using the positional Burrows-Wheeler transform (PBWT). Bioinformatics 30: 1266-1272.

Edmonds J, Johnson EL. 2003. Matching: a well-solved class of integer linear programs. In Combinatorial optimization-eureka, you shrink! (ed. (Jünger M, et al.), pp. 27-30. Springer, Berlin, Heidelberg.

Hach F, Sarrafi I, Hormozdiari F, Alkan C, Eichler EE, Sahinalp SC. 2014 mrsFAST-Ultra: a compact, SNP-aware mapper for high performance sequencing applications. Nucleic Acids Res 42(Web Server issue): W494-W500.

Haussler D, Smuga-Otto M, Paten B, Novak AM, Nikitin S, Zueva M, Miagkov D. 2017. A flow procedure for the linearization of genome sequence graphs. bioRxiv. doi: 10.1101/101501.

Herbig A, Jäger G, Battke F, Nieselt K. 2012. GenomeRing: alignment visualization based on SuperGenome coordinates. Bioinformatics 28: $7-15$.

Holley G, Peterlongo P. 2012. BlastGraph: intensive approximate pattern matching in string graphs and de-Bruijn graphs. In Proceedings of the Prague stringology conference 2012. Czech Technical University, Prague.

Huang L, Popic V, Batzoglou S. 2013. Short read alignment with populations of genomes. Bioinformatics 29: i361-i370.

International Human Genome Sequencing Consortium. 2001. Initial sequencing and analysis of the human genome. Nature 409: 860-921.

International Human Genome Sequencing Consortium. 2004. Finishing the euchromatic sequence of the human genome. Nature 431: 931-945.

Jain L. 2015. The future of personalized and precision perinatal medicine. Clin Perinatol 42: xvii-xix.

Jain M, Fiddes IT, Miga KH, Olsen HE, Paten B, Akeson M. 2015. Improved data analysis for the MinION nanopore sequencer. Nat Methods 12: 351-356.

Langmead B, Salzberg SL. 2012. Fast gapped-read alignment with Bowtie 2. Nat Methods 9: 357-359.

Lee C, Grasso C, Sharlow MF. 2002. Multiple sequence alignment using partial order graphs. Bioinformatics 18: 452-464.

Lee WP, Stromberg MP, Ward A, Stewart C, Garrison EP, Marth GT. 2014 MOSAIK: a hash-based algorithm for accurate next-generation sequencing short-read mapping. PLoS One 9: e90581.

Leonardsen EH. 2016. "Aligning reads against a graph based reference genome." Master's thesis, University of Oslo, Oslo, Norway.
Levy S, Sutton G, Ng PC, Feuk L, Halpern AL, Walenz BP, Axelrod N, Huang J, Kirkness EF, Denisov G, et al. 2007. The diploid genome sequence of an individual human. PLOS Biol 5: e254.

Li H. 2013. Aligning sequence reads, clone sequences and assembly contigs with BWA-MEM. arXiv:1303.3997.

Li N, Stephens M. 2013. Modeling linkage disequilibrium and identifying recombination hotspots using single-nucleotide polymorphism data. Genetics 165: 2213-2233.

Li H, Handsaker B, Wysoker A, Fennell T, Ruan J, Homer N, Marth G, Abecasis G, Durbin R. 2009. The Sequence Alignment/Map format and SAMtools. Bioinformatics 25: 2078-2079.

Limasset A, Cazaux B, Rivals E, Peterlongo P. 2016. Read mapping on de Bruijn graphs. BMC Bioinformatics 17: 237.

Liu B, Guo H, Brudno M, Wang Y. 2016. deBGA: read alignment with de Bruijn graph-based seed and extension. Bioinformatics 32: 3224-3232.

Maciuca S, del Ojo Elias C, McVean G, Iqbal Z. 2016. A natural encoding of genetic variation in a Burrows-Wheeler Transform to enable mapping and genome inference. In Algorithms in bioinformatics (ed. Frith M, Pedersen CN), pp. 222-233. Springer, Heidelberg, Germany.

Manuelidis L, Wu JC. 1978. Homology between human and simian repeated DNA. Nature 276: 92-94.

Medvedev P, Brudno M. 2009. Maximum likelihood genome assembly. J Comput Biol 16: 1101-1116.

Medvedev P, Stanciu M, Brudno M. 2009. Computational methods for discovering structural variation with next-generation sequencing. Nat Methods 6: S13-S20.

Miga KH. 2015. Completing the human genome: the progress and challenge of satellite DNA assembly. Chromosome Res 23: 421-426.

Miga KH, Newton Y, Jain M, Altemose N, Willard HF, Kent WJ. 2014. Centromere reference models for human chromosomes $\mathrm{X}$ and $\mathrm{Y}$ satellite arrays. Genome Res 24: 697-707.

Navarro A, Betrán E, Barbadilla A, Ruiz A. 1997. Recombination and gene flux caused by gene conversion and crossing over in inversion heterokaryotypes. Genetics 146: 695-709.

Need AC, Goldstein DB. 2009. Next generation disparities in human genomics: concerns and remedies. Trends Genet 25: 489-494.

Nguyen N, Hickey G, Zerbino DR, Raney B, Earl D, Armstrong J, Kent WJ Haussler D, Paten B. 2015. Building a pan-genome reference for a population. J Comput Biol 22: 387-401.

Nielsen R, Paul JS, Albrechtsen A, Song YS. 2011. Genotype and SNP calling from next-generation sequencing data. Nat Rev Genet 12: 443-451.

Novak AM, Rosen Y, Haussler D, Paten B. 2015. Canonical, stable, general mapping using context schemes. Bioinformatics 31: 3569-3576.

Novak AM, Garrison E, Paten B. 2016. A graph extension of the positional Burrows-Wheeler transform and its applications. In Algorithms in bioinformatics (ed. Frith M, Pedersen CN), pp. 246-256. Springer, Heidelberg, Germany.

Novak AM, Hickey G, Garrison E, Blum S, Connelly A, Dilthey A, Eizenga J, Elmohamed MAS, Guthrie S, Kahles A, et al. 2017. Genome graphs. bioRxiv. doi: 10.1101/101378.

Onodera T, Sadakane K, Shibuya T. 2013. Detecting superbubbles in assembly graphs. In Algorithms in bioinformatics (ed. Darling A, Stoye J), pp. 338-348. Springer, Heidelberg, Germany.

Paten B, Novak A, Haussler D. 2014. Mapping to a reference genome structure arXiv:1404.5010.

Paten B, Novak AM, Garrison E, Hickey G. 2017. Superbubbles, ultrabubbles and cacti. bioRxiv. doi: 10.1101/101493.

Pertea M, Kim D, Pertea GM, Leek JT, Salzberg SL. 2016. Transcript-level expression analysis of RNA-seq experiments with HISAT, StringTie and Ballgown. Nat Protoc 11: 1650-1667.

Petrovski S, Goldstein DB. 2016. Unequal representation of genetic variation across ancestry groups creates healthcare inequality in the application of precision medicine. Genome Biol 17: 157.

Pevzner PA, Tang H, Waterman MS. 2001. An Eulerian path approach to DNA fragment assembly. Proc Natl Acad Sci 98: 9748-9753.

Putnam NH, O'Connell BL, Stites JC, Rice BJ, Blanchette M, Calef R, Troll CJ Fields A, Hartley PD, Sugnet CW, et al. 2016. Chromosome-scale shotgun assembly using an in vitro method for long-range linkage. Genome Res 26: 342-350.

Rand KD, Grytten I, Nederbragt A, Storvik GO, Glad IK, Sandve GK. 2016 Coordinates and intervals in graph-based reference genomes. bioRxiv. doi: 10.1101/063206.

Rosen Y, Eizenga J, Paten B. 2017. Modelling haplotypes with respect to reference cohort variation graphs. bioRxiv. doi: 10.1101/101659.

Schneeberger K, Hagmann J, Ossowski S, Warthmann N, Gesing S, Kohlbacher O, Weigel D. 2009. Simultaneous alignment of short reads against multiple genomes. Genome Biol 10: R98.

Schneider VA, Graves-Lindsay T, Howe K, Bouk N, Chen HC, Kitts PA, Murphy TD, Pruitt KD, Thibaud-Nissen F, Albracht D, et al. 2017. Evaluation of GRCh38 and de novo haploid genome assemblies 
Paten et al.

demonstrates the enduring quality of the reference assembly. Genome Res (this issue). doi: 10.1101/gr.213611.116.

Seo JS, Rhie A, Kim J, Lee S, Sohn MH, Kim CU, Hastie A, Cao H, Yun JY, Kim J, et al. 2016. De novo assembly and phasing of a Korean human genome. Nature 538: 243-247.

Sherry ST, Ward MH, Kholodov M, Baker J, Phan L, Smigielski EM, Sirotkin K. 2001. dbSNP: the NCBI database of genetic variation. Nucleic Acids Res 29: 308-311.

Sirén J. 2017. Indexing variation graphs. In 2017 Proceedings of the ninteenth workshop on algorithm engineering and experiments (ALENEX). Society for Industrial and Applied Mathematics (SIAM), Barcelona, Spain.
Sudmant PH, Rausch T, Gardner EJ, Handsaker RE, Abyzov A, Huddleston J, Zhang Y, Ye K, Jun G, Hsi-Yang Fritz M, et al. 2015. An integrated map of structural variation in 2,504 human genomes. Nature 526: 75-81.

Willard HF, Waye JS. 1987. Hierarchical order in chromosome-specific human $\alpha$ satellite DNA. Trends Genet 3: 192-198.

Wu TD, Nacu S. 2010. Fast and SNP-tolerant detection of complex variants and splicing in short reads. Bioinformatics 26: 873-881.

Zerbino DR, Birney E. 2008. Velvet: algorithms for de novo short read assembly using de Bruijn graphs. Genome Res 18: 821-829.

\section{Genome Research}




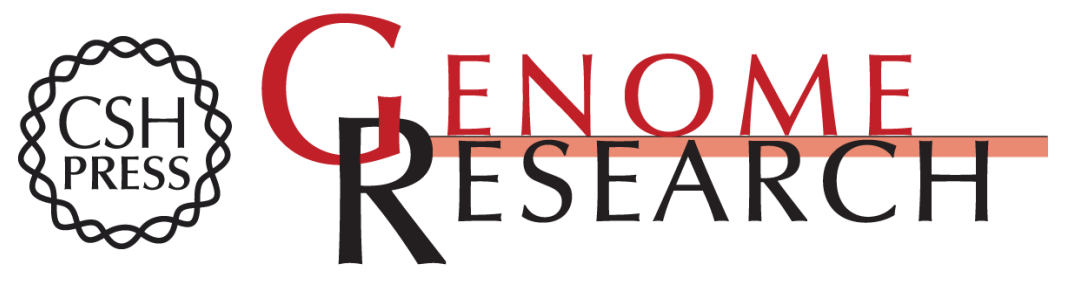

\section{Genome graphs and the evolution of genome inference}

Benedict Paten, Adam M. Novak, Jordan M. Eizenga, et al.

Genome Res. 2017 27: 665-676 originally published online March 30, 2017

Access the most recent version at doi:10.1101/gr.214155.116

References This article cites 53 articles, 11 of which can be accessed free at: http://genome.cshlp.org/content/27/5/665.full.html\#ref-list-1

Open Access Freely available online through the Genome Research Open Access option.

Creative This article, published in Genome Research, is available under a Creative Commons

License Commons License (Attribution 4.0 International), as described at http://creativecommons.org/licenses/by/4.0/.

Email Alerting Receive free email alerts when new articles cite this article - sign up in the box at the Service top right corner of the article or click here.

\section{Affordable, Accurate Sequencing.}

To subscribe to Genome Research go to: https://genome.cshlp.org/subscriptions 